\title{
Estudio cinético de la descomposición térmica del carbonato de Estroncio en el sistema $\mathrm{SrCO}_{3}-\mathrm{Al}_{2} \mathrm{O}_{3}-\mathrm{SrSO}_{4}$
}

\author{
J. A. RODRÍGUEZ G. ${ }^{1}$, J. TORRES T. ${ }^{1}$, J. M. ALMANZA R. ${ }^{1}$ \\ ${ }^{1}$ CINVESTAV-IPN, Unidad Saltillo. Carretera Saltillo-Monterrey Km. 13. \\ Saltillo, Coahuila, México.
}

\begin{abstract}
La cinética de descomposición térmica del carbonato de Estroncio $\left(\mathrm{SrCO}_{3}\right)$ en el sistema $\mathrm{SrCO}_{3}-\mathrm{Al}_{2} \mathrm{O}_{3}$ - $\mathrm{SrSO}_{4}$ se estudió por medio de análisis térmico gravimétrico (ATG), utilizando el método isotérmico. Se prepararon mezclas de polvos 3:3:1 molar de $\mathrm{SrCO}_{3}, \mathrm{Al}_{2} \mathrm{O}_{3}$ y $\mathrm{SrSO}_{4}$ grado reactivo, las cuales fueron homogenizadas y tratadas a temperaturas entre 750 hasta $1100^{\circ} \mathrm{C}$, por periodo de 4 horas. Adicionalmente se estudio el efecto del tiempo de activación mecánica del $\mathrm{SrCO}_{3}$ sobre la cinética de descomposición. Se obtuvieron curvas representativas de la pérdida de peso de las muestras al incrementar la temperatura, a partir de ellas se realizó el estudio cinético. Para el rango de temperatura de 750 a $900^{\circ} \mathrm{C}$, el mecanismo de reacción que rige el proceso de descomposición corresponde a una contracción geométrica mediante la frontera de grano $\left(R_{1.1}\right)$ con una energía de activación $\left(\mathrm{E}_{\mathrm{a}}\right)$ de $106.21 \mathrm{KJmol}^{-1}$. En el rango de temperatura de 950 a $1100^{\circ} \mathrm{C}$, el mecanismo de reacción que rige la descomposición corresponde a un proceso de nucleación y crecimiento $\left(\mathrm{P}_{11}\right)$, con un valor de $\mathrm{E}_{\mathrm{a}}$ de $44.87 \mathrm{KJmol}^{-1}$. La energía de activación disminuye conforme se incrementa el tiempo de activado mecánico hasta un 35\% para el caso de siete horas.
\end{abstract}

Palabras clave: cinética, análisis termo gravimétrico, método isotérmico, activación mecánica, energía de activación.

Kinetics of Thermal decomposition of strontium carbonate in the $\mathrm{SrCO}_{3}-\mathrm{Al}_{2} \mathrm{O}_{3}-\mathrm{SrSO}_{4}$ system

The thermal decomposition of strontium carbonate in the $\mathrm{SrCO}_{3}-\mathrm{Al}_{2} \mathrm{O}_{3}-\mathrm{SrSO}_{4}$ system was studied by thermal analysis under isothermal experiments. Powder of reactive grade of $\mathrm{SrCO}_{3}, \mathrm{Al}_{2} \mathrm{O}_{3}$ y $\mathrm{SrSO}_{4}$ in molar ratio 3:3:1 were prepared. The powders were heat treated from 750 to $1000{ }^{\circ} \mathrm{C}$ for $4 \mathrm{~h}$. Loss weight for each temperature was registered and the kinetics parameter were determine using the classical fit method. The effect of mechanical activation of $\mathrm{SrCO}_{3}$ was studied as well. The reaction mechanism for 750 to $900{ }^{\circ} \mathrm{C}$ temperature range corresponded to a geometric shrinkage in the grain boundary $\left(\mathrm{R}_{11}\right)$ with an activation energy of 106. $21 \mathrm{Kjmol}^{-1}$. The reaction mechanism for 900 to $1100{ }^{\circ} \mathrm{C}$ temperature range corresponded to a nucleation and growing $\left(\mathrm{P}_{1.1}\right)$ with an activation energy of $44.87 \mathrm{Kjmol}^{-1}$. The activation energy was reduced in 35\% for the samples that contained $\mathrm{SrCO}_{3}$ mechanically activated.

Keywords: kinetics, thermal gravimetric analysis, isothermal method. Mechanical activation, activation energy.

\section{INTRODUCCIÓN}

El Estroncio se encuentra en la naturaleza en forma de celestita $\left(\mathrm{SrSO}_{4}\right)$. Este mineral puede ser procesado para formar compuestos tales como carbonatos, nitratos y óxidos (1). El carbonato $\left(\mathrm{SrCO}_{3}\right)$ es el más importante de los compuestos de Estroncio, el cual tiene diversas aplicaciones, destacándose la producción de cinescopios para televisores a color (2), la preparación de materiales superconductores, en la industria del Acero y en la fundición de aleaciones de Aluminio (3).

El fundamento de la descomposición de este carbonato y el conocimiento tanto de los parámetros cinéticos como del mecanismo de reacción, resulta esencial cuando se desea formar diversos compuestos. La descomposición térmica involucra transporte de gas, formación de capa de óxido, reacción química y transferencia de calor. Esta comienza en la superficie de la partícula hacia el interior de la misma.

El modelo cinético y los parámetros derivados del análisis térmico dependen de las mediciones y condiciones de la muestra. Si ambos (modelo y parámetros), difieren bajo varias condiciones experimentales, estos cambios son apreciables en variaciones en los mecanismos de reacción (4). Se han realizado estudios relacionados con la descomposición térmica de carbonatos de metales alcalinotérreos ( $\mathrm{Ca}, \mathrm{Sr}$ y $\mathrm{Ba}$ ), sin embargo, las condiciones experimentales y los resultados, en cada caso varían de sistema a sistema. Boris (5) reporta valores de $\mathrm{E}_{\mathrm{a}}$ correspondientes a la descomposición del $\mathrm{SrCO}_{3}$ en polvo desde temperatura ambiente hasta $1000^{\circ} \mathrm{C}$, obteniendo un valor de $\mathrm{E}_{\mathrm{a}}$ : $286 \mathrm{KJmol}^{-1}$. Sin embargo no se menciona el mecanismo cinético que rige dicho proceso (método dinámico). Arvanitidis (3) reporta un valor de $\mathrm{E}_{\mathrm{a}}$ de $267 \mathrm{KJmol}^{-1}$ para la descomposición del $\mathrm{SrCO}_{3}$ (en forma de polvo) con un mecanismo cinético $\mathrm{F}_{1}$. Maitra (6) reporta un valor de $\mathrm{E}_{\mathrm{a}}$ de $238.22 \mathrm{KJmol}^{-1}$ (muestra en polvo) con un mecanismo cinético $\mathrm{D}_{4}$. Nobuyoshi (4) reporta la $\mathrm{E}_{\mathrm{a}}$ de $218 \mathrm{KJmol}^{-1}$ para la descomposición del $\mathrm{SrCO}_{3}$ en muestras en forma de polvos con un mecanismo cinético $R_{1.2}$.

La cinética de descomposición de carbonatos alcalinotérreos ha sido de gran interés desde hace tiempo, sin embargo los mecanismos cinéticos presentan variaciones, lo cual puede ser 
indicativo de las condiciones experimentales y tipo de sistema estudiado tiene una gran influencia.

El objetivo de este trabajo es llevar acabo el estudio cinético de la descomposición térmica del $\mathrm{SrCO}_{3}$ a $\mathrm{SrO}$ en el sistema $\mathrm{SrCO}_{3}-\mathrm{Al}_{2} \mathrm{O}_{3}-\mathrm{SrSO}_{4^{\prime}}$ a partir del análisis térmico gravimétrico (ATG) realizado a muestras compactadas bajo condiciones isotérmicas.

\section{EXPERIMENTACIÓN}

\subsection{Preparación de las muestras.}

Se mezclaron los reactivos de grado químico $\mathrm{SrCO}_{3^{\prime}} \mathrm{Al}_{2} \mathrm{O}_{3}$ y $\mathrm{SrSO}_{4}$ de acuerdo a la relación molar 3:3:1. La mezcla se colocó dentro de un recipiente cilíndrico con acetona y bolas de Alúmina y se homogenizó en rodillos giratorios. La mezcla se secó en una mufla a $60^{\circ} \mathrm{C}$ durante 24 horas y se molió en un mortero para la eliminación de aglomerados. Se conformaron pastillas de $5 \mathrm{~mm}$ de espesor por prensado uniaxial a $100 \mathrm{MPa}$, con un peso aproximado de $100 \mathrm{mg}$.

Para el estudio cinético, las muestras se analizaron por medio de análisis termo-gravimétrico, utilizando el método isotérmico en el rango de temperatura de $750-1100^{\circ} \mathrm{C}$, a una velocidad de calentamiento de $10^{\circ} \mathrm{Cmin}^{-1} \mathrm{y}$ mantenimiento por cuatro horas. Se obtuvieron curvas representativas de la pérdida de peso de las muestras contra la temperatura.

Adicionalmente se estudio el efecto de la activación mecánica sobre la cinética de descomposición del $\mathrm{SrCO}_{3}$ en el sistema $\mathrm{SrCO}_{3}-\mathrm{Al}_{2} \mathrm{O}_{3}-\mathrm{SrSO}_{4}$. Muestras de carbonato de Estroncio fueron activadas mecánicamente en un molino planetario (Retsch PM 200) durante 3, 5 y 7 horas. Se prepararon mezclas de los tres reactivos con el material activado de acuerdo al procedimiento descrito anteriormente. El estudio cinético isotérmico se realizó a las mismas condiciones que para la mezcla sin activación mecánica.

\subsection{Interpretación de resultados}

El análisis cinético se realizó siguiendo el procedimiento clásico con el objetivo de determinar la ecuación de velocidad que describa la formación de productos a determinados tiempos, usualmente pero no necesariamente, a temperatura constante (mecanismo de reacción) y la determinación de la influencia de la temperatura sobre la velocidad de reacción. La dependencia de la temperatura sobre la energía de activación se expresa por la Ecuación de Arrhenius:

$$
k=A \exp \left(\frac{-E_{a}}{R T}\right)
$$

Donde $\mathrm{R}$ es la constante de los gases, $\mathrm{T}$ es la temperatura, $\mathrm{E}_{\mathrm{a}}$ es la Energía de activación y A: Factor de frecuencia.

La fracción reaccionada $(\alpha)$ en los experimentos se determinó de acuerdo a la Ecuación 2.

$$
\alpha=\frac{\left(m_{0}-m_{t}\right)}{\left(m_{0}-m_{f}\right)}
$$

Donde $\mathrm{m}_{\mathrm{o}}$ es la masa inicial, $\mathrm{m}_{\mathrm{t}}$ es la pérdida o ganancia de masa al tiempo t y $\mathrm{m}_{\mathrm{f}}$ es la pérdida ó ganancia de masa total.
El siguiente paso en el estudio cinético es graficar la fracción reaccionada contra el tiempo para todo el rango de temperatura. El valor de la fracción reaccionada es evaluado en los diferentes modelos cinéticos para reacciones en estado sólido (7), determinándose de esa manera la función $g(\alpha)$. Se grafica $g(\alpha)$ contra el tiempo para cada modelo cinético y se comparan con las curvas de fracción reaccionada identificándose cual modelo presenta mayor semejanza entre sus curvas con respecto a las de la fracción reaccionada determinándose de esa manera el mecanismo cinético que rige el proceso de descomposición del carbonato de Estroncio.

Al aplicar ln a la Ecuación 1 nos queda:

$$
\ln \mathrm{k}=\left[\left(\frac{-\mathrm{E}_{\mathrm{a}}}{\mathrm{R}}\right)\left(\frac{1}{\mathrm{~T}}\right)\right]+\ln \mathrm{A}
$$

Graficando ln k contra 1/T para cada temperatura se puede realizar una regresión lineal entre los puntos cuyo valor de la pendiente $(\mathrm{m})$ es equivalente $\mathrm{a}-\mathrm{E}_{\mathrm{a}} / \mathrm{R}$ (Ecuación de la recta).

\section{RESULTADOS Y DISCUSIÓN}

En la Figura 1 se presentan las curvas correspondientes a la fracción reaccionada durante el proceso de descomposición del $\mathrm{SrCO}_{3}$ (en la mezcla) analizada a diferentes temperaturas sin activación mecánica.

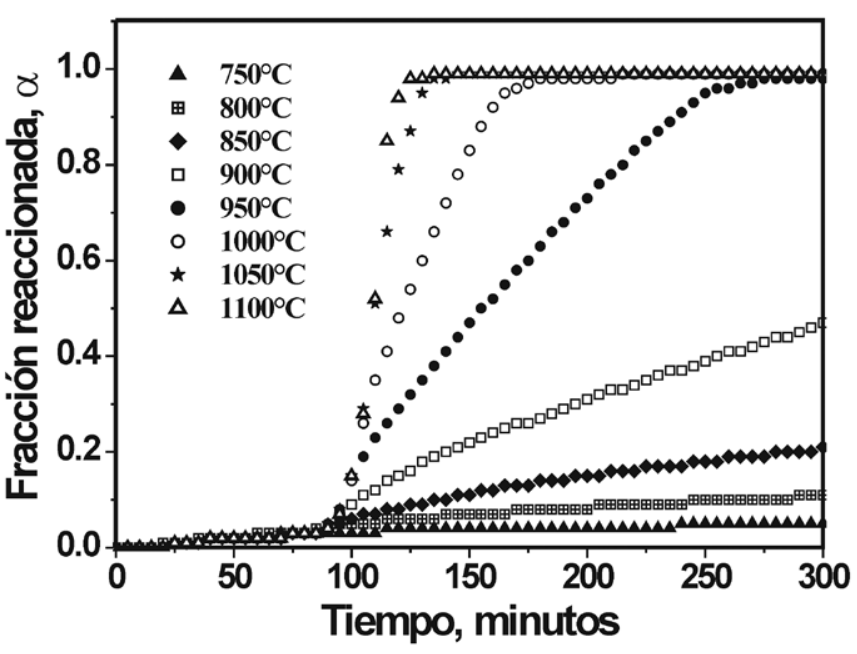

Fig. 1- Curvas de fracción reaccionada $\alpha$ contra el tiempo correspondientes a la descomposición térmica del $\mathrm{SrCO}_{3}$ en el sistema $\mathrm{SrCO}_{3}-\mathrm{Al}_{2} \mathrm{O}_{3}-\mathrm{SrSO}_{4}$ sin activación mecánica.

Se puede observar el incremento en la fracción reaccionada conforme aumenta la temperatura, hasta llegar muy cerca de la unidad (muestras analizadas en el rango de $950-1100^{\circ} \mathrm{C}$ ).

Se encontró que existen dos modelos cinéticos que se ajustan al proceso de descomposición en este sistema, el $\mathrm{R}_{1.1}$ (contracción geométrica mediante la frontera de grano, $\left.1-(1-\alpha)^{1 / n}\right)$ y $P_{1.1}$ (nucleación y crecimiento mediante la ley de energía, $\left.\alpha^{1 / n}\right)$. Estos resultados son similares a los reportados 
por Nobuyoshy (4) para la descomposición de carbonatos alcalinotérreos. Las gráficas comparativas se presentan a continuación.

El valor de $\mathrm{n}$ en estos dos modelos cinéticos se varió en el rango entre 1 y 3 , resultando el valor de 1.1 con la mejor correlación entre las curvas. Las curvas tanto para $\mathrm{R}_{11}$ como para $\mathrm{P}_{1.1}$ presentan buena semejanza entre sus valores, sin embargo se puede apreciar que para el caso del modelo $R_{1.1}$ existe mayor ajuste entre sus curvas para bajas temperaturas $\left(750-900^{\circ} \mathrm{C}\right)$, caso contrario para el modelo cinético $P_{1.1}$ que presenta mayor ajuste entre las curvas correspondientes a temperaturas elevadas $\left(950-1100^{\circ} \mathrm{C}\right)$.
Los valores de la Energía de activación determinados a partir del método de ajuste de las funciones, se presentan en la Tabla I.

El proceso de descomposición del $\mathrm{SrCO}_{3}$ en el sistema $\mathrm{SrCO}_{3}-\mathrm{Al}_{2} \mathrm{O}_{3}-\mathrm{SrSO}_{4}$, a bajas temperaturas $\left(750-900^{\circ} \mathrm{C}\right)$ sufre una rápida y densa nucleación inicial a través de todas o algunas fases cristalinas, debido a la formación de una zona de interfase de reacción (a valores bajos de $\alpha$ ), como consecuencia de la descomposición del $\mathrm{SrCO}_{3}$ en el volumen de la muestra, lo que genera una producción masiva de núcleos a velocidad constante, en ausencia de efectos difusivos y sin avanzar mas allá del cristal. A temperaturas elevadas $\left(950-1100^{\circ} \mathrm{C}\right)$, se
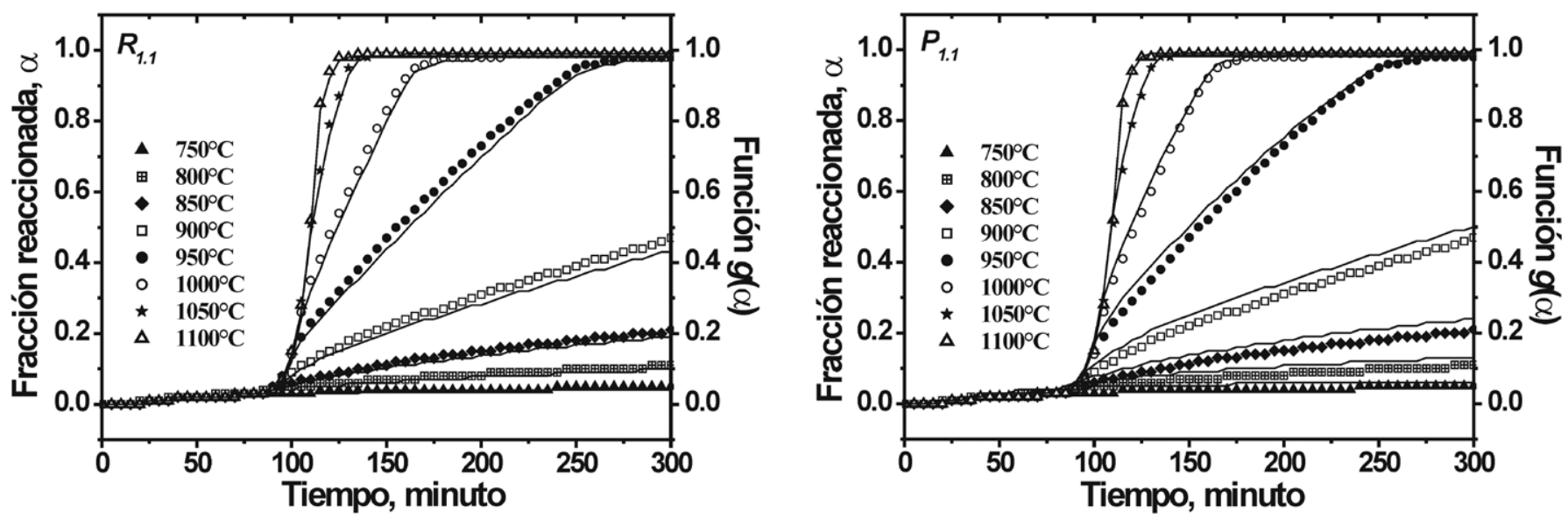

Fig. 2- Correlación que existe entre las curvas de los modelos cinéticos $\mathrm{R}_{1.1}$ y $\mathrm{P}_{1.1}$ con las curvas representativas de la fracción reaccionada.

Ambos modelos cinéticos rigen la descomposición del $\mathrm{SrCO}_{3}$ en el sistema $\mathrm{SrCO}_{3}-\mathrm{Al}_{2} \mathrm{O}_{3}-\mathrm{SrSO}_{4}$, tomando en cuenta que el estudio cinético de un proceso es un método de aproximación de los fenómenos que están ocurriendo.

De esta manera la Energía de activación se determinó para cada modelo en el rango de temperatura correspondiente. Las pendientes de Arrhenius donde se determinaron los valores de energía de activación se muestran en la Figura 3.

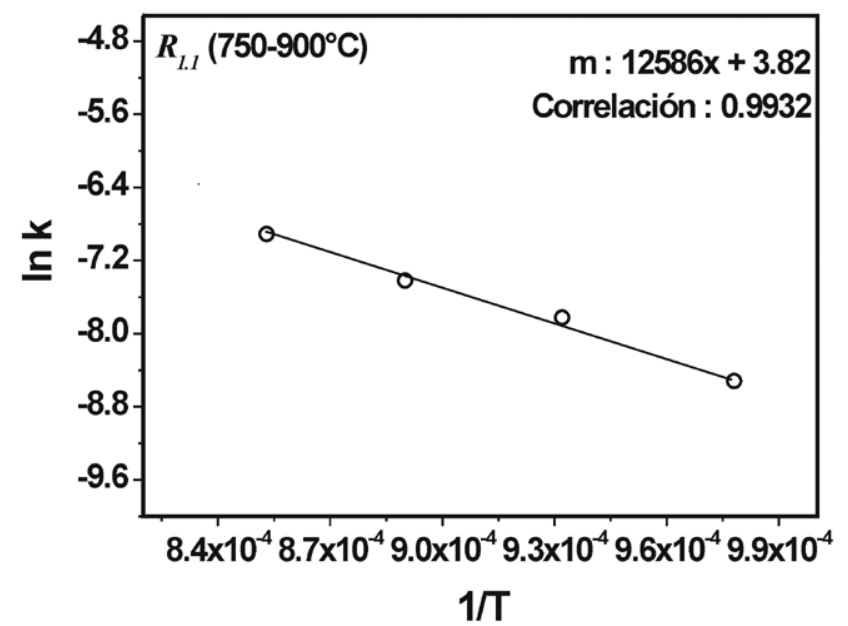

incrementan las zonas de reacción (interfase activa) y por lo consiguiente se desarrollan una mayor cantidad de núcleos, conjuntamente con el crecimiento de los mismos formando el volumen total del producto.

Los resultados de los experimentos cinéticos utilizando muestras con carbonato de Estroncio activado mecánicamente presentaron comportamiento similar a las muestras con $\mathrm{SrCO}_{3}$ sin activar en cuanto a los modelos cinéticos que rigen

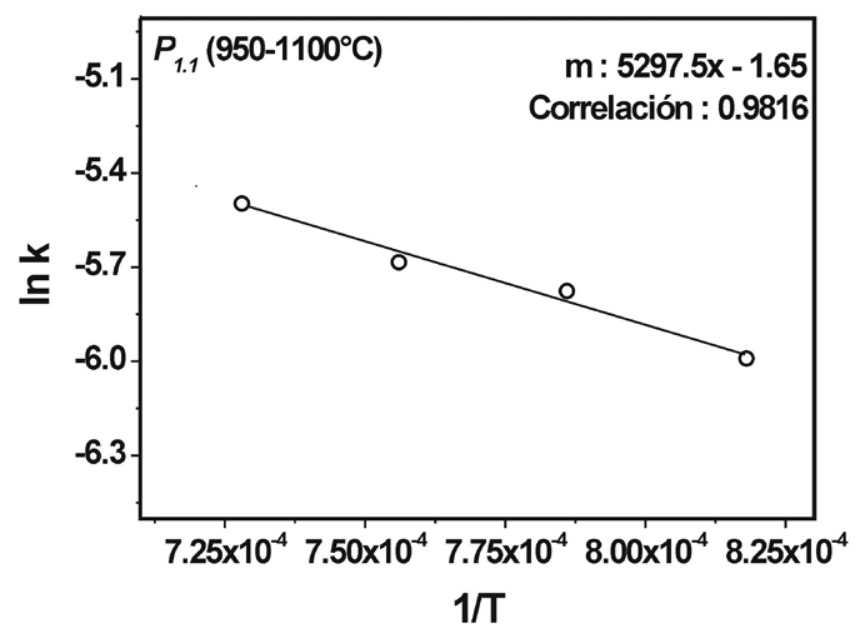

Fig. 3- Determinación de la pendiente de Arrhenius correspondiente a los dos mecanismos de reacción en sus respectivos rangos de temperatura y sus correlaciones. 
TABLA I. E OBTENIDOS MEDIANTE LOS DOS MECANISMOS DE REACCIÓN PARA LA DESCOMPOSICIÓN DEL SRCO EN EL SISTEMA SRCO $-\mathrm{AL}_{2} \mathrm{O}_{3}-\mathrm{SRSO}_{4}$

\begin{tabular}{|c|c|c|c|}
\hline Modelo cinético & Temperatura, ${ }^{\circ} \mathrm{C}$ & $\mathrm{E}_{\mathrm{a}^{\prime}} \mathrm{KJmol}^{-1}$ & $\begin{array}{c}\text { Método de } \\
\text { determinación }\end{array}$ \\
\hline $\mathrm{R}_{1.1}$ & $750-900$ & 106.21 & $\begin{array}{l}\text { Ajuste de } \\
\text { funciones }\end{array}$ \\
\hline $\mathrm{P}_{1.1}$ & $950-1100$ & 44.87 & $\begin{array}{c}\text { Ajuste de } \\
\text { funciones }\end{array}$ \\
\hline
\end{tabular}

TABLA II. ENERGÍA DE ACTIVACIÓN DE LAS MUESTRAS ACTIVADAS MECÁNICAMENTE

\begin{tabular}{|c|cc|cc|}
\hline \multirow{2}{*}{$\begin{array}{c}\text { Periodo de } \\
\text { activación, } \mathrm{h}\end{array}$} & \multicolumn{4}{|c|}{ Rango de temperatura, ${ }^{\circ} \mathrm{C}$} \\
\cline { 2 - 5 } & $750-900$ & $\left(\mathrm{R}_{1.1}\right)$ & $950-1100$ & $\left(\mathrm{P}_{1.1}\right)$ \\
\hline 3 & 83.32 & $(0.9942)$ & 40.30 & $(0.9940)$ \\
\hline 5 & 57.56 & $(0.9840)$ & 33.53 & $(0.9840)$ \\
\hline 7 & 47.09 & $(0.9885)$ & 29.45 & $(0.9776)$ \\
\hline
\end{tabular}

el proceso de descomposición se refiere. Los mecanismos de reacción que rigen el proceso para los tres tiempos de activación, son de igual forma el $\mathrm{R}_{1.1}$ para bajas temperaturas $\left(750-900^{\circ} \mathrm{C}\right)$ y el $\mathrm{P}_{1.1}$ para temperaturas elevadas $\left(950-1100^{\circ} \mathrm{C}\right)$ y los valores de la Energía de activación determinados por el método de ajuste de funciones se presentan en la Tabla II.

El efecto de la activación mecánica es claro. Al incrementar el tiempo de activación se reduce la $\mathrm{E}_{\mathrm{a}}$ necesaria para la descomposición del $\mathrm{SrCO}_{3}$ en este sistema, efecto que ya ha sido reportado en la literatura (8).

De los resultados anteriores se puede observar que las energías de activación en este trabajo son mucho menores a las reportadas en la literatura, esto puede deberse a que la muestra que se analizó fue compactada a $100 \mathrm{MPa}$, ya que se ha reportado que al incremento en la compactación de carbonatos disminuye la $\mathrm{E}_{\mathrm{a}}$ (9). Adicionalmente los experimentos de cinética se realizaron para un sistema ternario, por consiguiente se espera que el $\mathrm{SrO}$ reaccione con la $\mathrm{Al}_{2} \mathrm{O}_{3}$ y el $\mathrm{SrSO}_{4}$ para formar compuestos de Estroncio. Para corroborar dicha afirmación se realizaron análisis de DRX y MEB en muestras de la mezcla $\mathrm{SrCO}_{3}-\mathrm{Al}_{2} \mathrm{O}_{3}-\mathrm{SrSO}_{4}$ tratadas térmicamente de 800 a $1150^{\circ} \mathrm{C}$. La Figura 4 presenta los resultados obtenidos de difracción de rayos $X$.

Se puede observar que a $800^{\circ} \mathrm{C}$ se detecta la presencia de los reactivos iniciales más una pequeña cantidad de un compuesto de Estroncio, el $\mathrm{Sr}_{3} \mathrm{Al}_{2} \mathrm{O}_{6}$. Al incrementar la temperatura $\left(900^{\circ} \mathrm{C}\right)$, la cantidad del aluminato de Estroncio también aumenta, al mismo tiempo que se comienza a formarse la fase $\mathrm{Sr}_{4} \mathrm{Al}_{6} \mathrm{O}_{12} \mathrm{SO}_{4}$. En la muestra sinterizada a $1000^{\circ} \mathrm{C}$ se observa la intensificación de los picos correspondientes a los dos compuestos de Estroncio registrados y la formación de un segundo aluminato de Estroncio $\left(\mathrm{SrAl}_{2} \mathrm{O}_{4}\right)$. Conforme se incrementa la temperatura $\left(1100^{\circ} \mathrm{C}\right)$ la intensidad de los picos de los dos aluminatos de Estroncio decrece y finalmente a

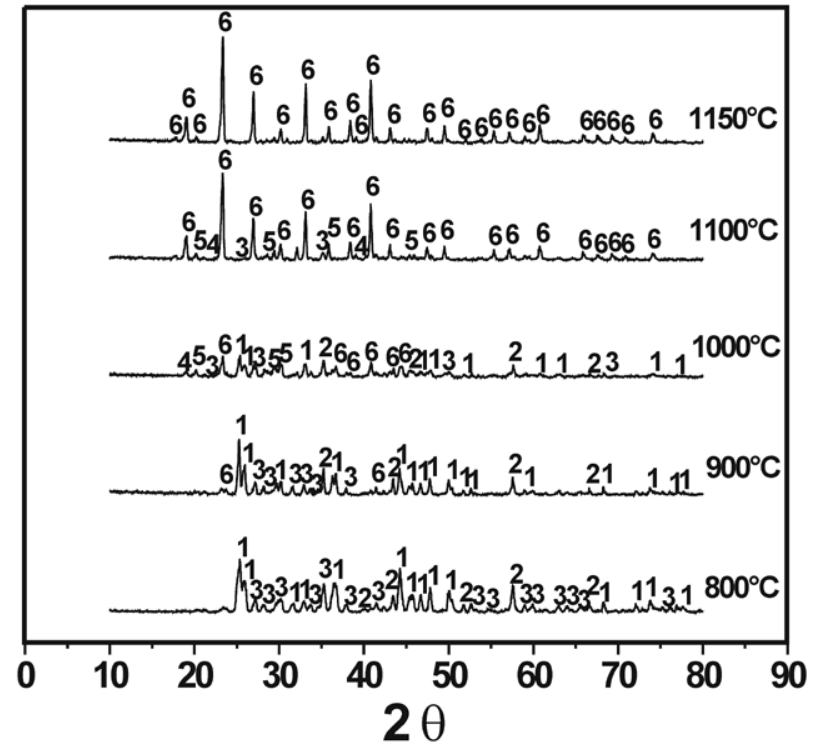

Fig. 4- Patrones de difracción de rayos $\mathrm{X}$ de muestras sinterizadas de 800 a $1150^{\circ} \mathrm{C}$ por 6 horas. 1: $\mathrm{SrCO}_{3^{\prime}} 2: \mathrm{Al}_{2} \mathrm{O}_{3^{\prime}}, 3: \mathrm{SrSO}_{4^{\prime}} 4: \mathrm{Sr}_{3} \mathrm{Al}_{2} \mathrm{O}_{6^{\prime}}$;: $\mathrm{SrAl}_{2} \mathrm{O}_{4}$ and 6: $\mathrm{Sr}_{4} \mathrm{Al}_{6} \mathrm{O}_{12} \mathrm{SO}_{4}$.
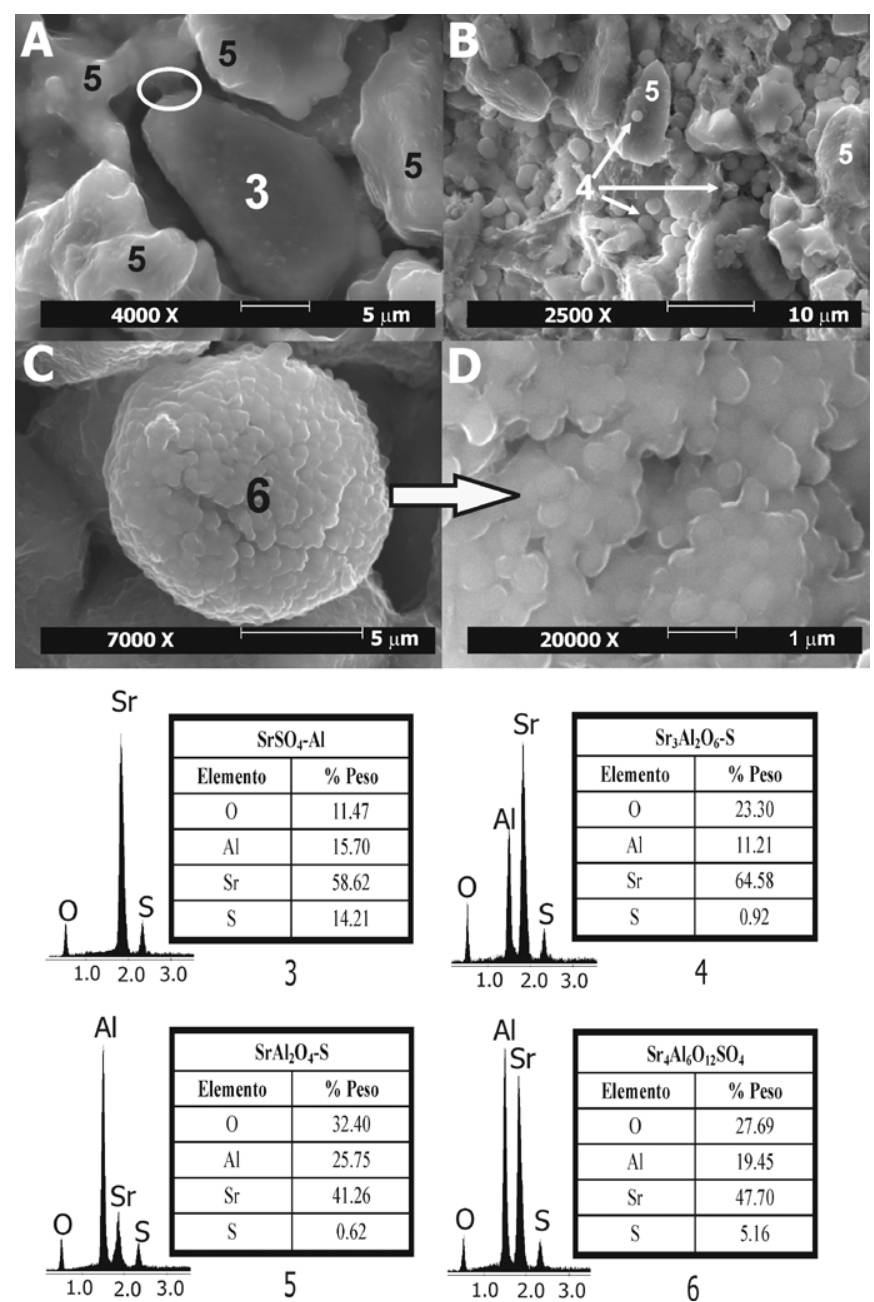

Fig. 5- Microfotografías de muestras sinterizadas a 1000 y $1100^{\circ} \mathrm{C}$ por 4 y 6 horas respectivamente. Se presentan las fases encontradas en cada muestra, correspondientes a las reportadas en DRX. A: $1000^{\circ} \mathrm{C}-4 \mathrm{~h}, \mathrm{~B}$ : $1000^{\circ} \mathrm{C}-6 \mathrm{~h}, \mathrm{C}$ y D: $1100^{\circ} \mathrm{C}-6 \mathrm{~h}$. 
$1150^{\circ} \mathrm{C}$ se registra únicamente la presencia del sulfualuminato de Estroncio $\mathrm{Sr}_{4} \mathrm{Al}_{6} \mathrm{O}_{12} \mathrm{SO}_{4}$.

En los patrones de difracción de la Figura 4, se puede observar que no se registra los picos correspondientes a el $\mathrm{SrO}$ a ninguna temperatura, esto puede relacionarse con una reacción instantánea del óxido con la Alúmina y el sulfato de Estroncio presentes en el sistema formándose compuestos de Estroncio $\left(\mathrm{Sr}_{3} \mathrm{Al}_{2} \mathrm{O}_{6}, \mathrm{SrAl}_{2} \mathrm{O}_{4}\right.$ y $\left.\mathrm{Sr}_{4} \mathrm{Al}_{6} \mathrm{O}_{12} \mathrm{SO}_{4}\right)$. Por otro lado, el $\mathrm{CO}_{2}$ producido durante el proceso de descomposición del carbonato se transporta a través de las capas de reacción por medio de procesos difusivo, lo cual no se tiene establecido el efecto del mismo en la velocidad de reacción.

Se realizaron estudios de microscopía electrónica de barrido a muestras (de la mezcla) sinterizadas a diferentes temperaturas y tiempos, con el fin de encontrar las fases reportadas en DRX y corroborar los resultados. La Figura 5 presenta microfotografías correspondientes a muestras sinterizadas a 1000 y $1100^{\circ} \mathrm{C}$ durante 4 y 6 horas, respectivamente.

En la Figura 5a se puede observar la interacción entre una partícula de sulfato de Estroncio y las partículas correspondientes al aluminato de Estroncio $\mathrm{SrAl}_{2} \mathrm{O}_{4^{\prime}}$ así como una pequeña zona de interfase (zona donde se generan reacciones químicas) en forma de cuello. Para el caso de la Figura $5 \mathrm{~b}$ se presentan partículas esféricas correspondientes al aluminato de Estroncio $\mathrm{Sr}_{3} \mathrm{Al}_{2} \mathrm{O}_{6}$ que se encuentra en contacto con partículas irregulares $\left(\mathrm{SrAl}_{2} \mathrm{O}_{4}\right)$, de acuerdo a los análisis de EDS. En la Figura 5c se presenta una partícula esférica compuesta por aglomerados de pequeñas partículas pertenecientes al $\mathrm{Sr}_{4} \mathrm{Al}_{6} \mathrm{O}_{12} \mathrm{SO}_{4^{\prime}}$ la imagen $5 \mathrm{~d}$ corresponde a la misma partícula vista a mayores aumentos. De esta manera la formación de aluminatos de Estroncio ocurre de manera simultánea con la descomposición del $\mathrm{SrCO}_{3^{\prime}}$ el efecto de la formación de estos compuestos sobre la cinética de descomposición no es claro y merece un estudio mas profundo.

\section{CONCLUSIONES}

La descomposición del carbonato de Estroncio en el sistema $\mathrm{SrCO}_{3}-\mathrm{Al}_{2} \mathrm{O}_{3}-\mathrm{SrSO}_{4^{\prime}}$ de temperatura ambiente hasta $1100^{\circ} \mathrm{C}$, a $10^{\circ} \mathrm{Cmin}^{-1}$ se rige mediante dos mecanismos de reacción. De 750 a $900^{\circ} \mathrm{C}$ el proceso se lleva acabo mediante el mecanismo de contracción geométrica controlado por la frontera de grano $\left(\mathrm{R}_{1.1}\right)$, con una energía de activación de $106.21 \mathrm{KJmol}^{-1}$. Para el resto de las temperaturas $\left(950-1100^{\circ} \mathrm{C}\right)$, el proceso se rige por el mecanismo de nucleación y crecimiento mediante la ley de energía $\left(\mathrm{P}_{1.1}\right)$, con un valor de energía de activación de $44.87 \mathrm{KJmol}^{-1}$.

$\mathrm{El} \mathrm{SrO}$ generado es altamente reactivo y en presencia de $\mathrm{Al}_{2} \mathrm{O}_{3}$ y $\mathrm{SrSO}_{4}$, reacciona para formar nuevos productos. Estos compuestos se detectaron mediante las técnicas de MEB y DRX. El $\mathrm{CO}_{2}$ generado difunde a través de una capa de reacción.

Las muestras (mezcla) con carbonato de Estroncio mecánicamente activado, para los tres tiempos (3, 5 y 7 horas) presentaron el mismo comportamiento cinético, los mismos mecanismos de reacción rigen el proceso. Se puede observar el efecto de la activación mecánica en los resultados de los parámetros cinéticos, a mayor tiempo de activado, se reduce el valor de la energía de activación hasta un 35\% para el caso de las muestras activadas durante 7 horas.

\section{BIBLIOGRAFÍA}

1. M. J. Duran, Informe del grupo de productores de no metálicos, Minería CAMIMEX. V., 9, 8, 10-11 (1997).

2. J. Griffihts, Celestite: New production and processing developments, Ind. Min., 21-35 (1985).

3. I. Arvanitidis, D. Sichen, H. Y. Shon, S. Seetharama. The intrinsic thermal decomposition kinetics of $\mathrm{SrCO}_{3}$ by nonisothermal technique. Met. Mat. Trans. B. V., 28B, 1063-1068 (1997).

4. N. Koga, H. Tanaka, Kinetics of thermal decomposition of $\mathrm{MCO}_{3}$ to $\mathrm{MO}(\mathrm{M}$ = Ca, Sr and Ba), J. Ther. Anal. V., 34, 177-188 (1988).

5. B. V. L'vov, Mechanism and kinetics of thermal decomposition or carbonates. Thermochim. Acta. V., 386, 1-16 (2002).

6. S. Maitra, N. Banyopadhyay, S. Das, Non-isothermal decomposition kinetic of alkaline-earth metal carbonates, J. Am. Ceram. Soc. V., 90, 4, 1299-1303 (2007).

7. M. E. Brown, Handbook of thermal analysis and calorimetry. Ed. P. K. Gallagher Department of chemistry Ohio State University USA, Elsevier Science B.V. V., 1, 147-216 (1998).

8. J. M. Criado, F. González, J. Morales, Alteration of kinetics and thermodynamics of thermal decomposition of alkaline-earth carbonates induced by grinding, Thermochim. Acta. V., 32, 99-110 (1979).

9. S. Maitra, A. Choudhury, H. S. Das, MS. J. Pramanik, Effect of compaction on the kinetics of thermal decomposition of dolomite under non-isothermal condition, J. Mater. Sci. .V., 40, 4749-4751 (2005). 